\title{
RESCUE MANAGEMENT AND ASSESSMENT OF STRUCTURAL DAMAGE BY UAV IN POST-SEISMIC EMERGENCY
}

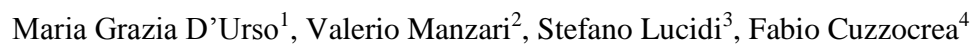 \\ ${ }^{1}$ DISA, Department of Engineering and Applied Sciences, University of Bergamo \\ Viale G. Marconi, 5 Dalmine (Bergamo), Italy \\ mariagrazia.durso@unibg.it \\ ${ }^{2}$ DICeM, Department of Civil and Mechanical Engineering, University of Cassino and Southern Lazio, \\ Via G. Di Biasio 43, Cassino (Frosinone), Italy \\ valerio.manzari@unicas.it \\ ${ }^{3}$ Direction Fire Department of Frosinone, Italy stefano.lucidi@ vigilfuoco.it \\ ${ }^{4}$ Regional Direction and Supervision of Fire Department of Calabria district, Italy \\ fabio.cuzzocrea@vigilfuoco.it
}

Commission V, WG 2

KEYWORDS: Rescue, control, seismic, UAV, damage, emergency, earthquake

\begin{abstract}
The increasing frequency of emergencies urges the need for a detailed and thorough knowledge of the landscape. The first hours after a disaster are not only chaotic and problematic, but also decisive to successfully save lives and reduce damage to the building stock. One of the most important factors in any emergency response is to get an adequate awareness of the real situation, what is only possible after a thorough analysis of all the available information obtained through the Italian protocol Topography Applied to Rescue. To this purpose geomatic tools are perfectly suited to create, manage and dynamically enrich an organized archive of data to have a quick and functional access to information useful for several types of analysis, helping to develop solutions to manage the emergency and improving the success of rescue operations. Moreover, during an emergency like an earthquake, the conventional inspection to assess the damage status of buildings requires special tools and a lot of time. Therefore, given the large number of buildings requiring safety measures and rehabilitation, efficient use of limited resources such as time and equipment, as well as the safety of the involved personnel are important aspects. The applications shown in the paper are intended to underline how the abovementioned objective, in particular the rehabilitation interventions of the built heritage, can be achieved through the use of data acquired from UAV platform integrated with geographic data stored in GIS platforms.
\end{abstract}

\section{INTRODUCTION}

Over the past few decades, natural phenomena that have struck our planet have progressively assumed the characteristics of extreme events, causing various problems in the management of the territory. Therefore, the scenarios caused by these types of events can often be classified as extreme and dangerous environments. In this context of continuous and unpredictable changes, the institutions involved in the land management have successfully experienced new technical tools put forth by the technological progress. Their use entails the need to get a growing spectrum of skills in different fields since the accumulation of data or the use of state-of-the-art technical tools are not sufficient by themselves to take timely and effective decisions concerning management problems of the territory without the presence of experienced personnel who can integrate and, above all, interpret the collected information. Hence, applications put forth by Geomatics, nowadays particularly efficient and suitable to describe natural hazards can help to share, compare and exchange data in a correct and accessible way, possibly following coded standards for the production of maps and user-friendly technologies for communicating results. The simplicity, precision and speed of field detection techniques are some of the ingredients that allow for better results. In this perspective, a key factor offered by outdoor digital techniques is the ability to organize a complete data set during field activities, thus avoiding lengthy laboratory operations such as copying data from paper forms (Garden et al. 2012). From a purely operational point of view, the recent disasters (from the L'Aquila earthquake in 2009 to the one in Mexico in 2017) have clearly demonstrated the potential role of Geomatics in supporting prompt actions in emergency situations and recovery, highlighting how geo-referencing information plays a crucial role in facilitating emergency management. Usually, depending on the scale of the disaster, several organizations are involved in initial response operations, such as, for example, the Fire Department, Police, humanitarian organisations and those of volunteers. Each of them relies on well-defined standard procedures that do not involve the exchange of information. In fact, although several methods have been developed to facilitate operations during an emergency response or a difficult event, a complete and shared inventory of the information collected by all agencies is still missing. In the areas struck by an earthquake, after the first missions by the Fire and Civil Protection for life rescue and the recovery of bodies, the first priority is the urgency to intervene with expeditive surveys for the assessment of damage and accessibility conditions in order to plan safety measures and consolidation of structures/infrastructures. The purpose of this work is to show how integrated techniques, specifically unmanned aerial vehicles (UAV) platforms, satellite photogrammetry, use of 
Global Network Satellite Systems (GNSS) and GIS systems can be of great support in the management of an emergency, allowing for the acquisition and storage of a large amount of data useful to perform context and detail analyses in areas struck by extreme and/or catastrophic events (D'Urso et alii, 2018). In particular, the detailed analysis carried out in this work aims at an innovation: the compilation of the TriageEdEm (Triage of The Building in Emergency) card, in use as ordinary facility at the Fire Brigades in Italy, by means of images captured by UAV in order to, greatly reduce rescue time and increasing the level of safety of the involved operators. These detection activities in extreme and inaccessible conditions have been conducted and tested for the first time with reference to residential buildings affected by the earthquake that struck the Lacco Ameno resort in the island of Ischia in 2017. Some real survey cases, aimed at carrying out immediate static consolidation and safety measures are also examined.

\section{PROTOCOLS OF INTERNATIONAL EMERGENCY MANAGEMENT}

The management of crisis situations, both natural and anthropic, requires a precise knowledge of the territory and of the relevant existing structures. Therefore it is necessary that all organizations / institutions that create or manage geospatial data, in any form or modality, work together in close coordination; in this way the amount of data can be produced and distributed to help the management of "ordinary" situations and the preparation of properly managed crisis situations. Hence helping institutions in charge of attending on the territory in a situation of difficulty or emergency is an absolute priority to avoid loss of life as much as possible (Santoro, 2017). For example, focusing on the initial stage of an emergency, which corresponds to the hours immediately following the occurrence of disastrous events, several organizations do activate. Among them is the International Charter "Space and Major Catastrophes" which aims to provide a unified system of acquisition and delivery of spatial data through authorized users to those who have been struck by natural or artificial disasters. The International Charter "Space and Major Catastrophes" manages information of all types concerning natural and artificial disasters in the world. This is possible by means of the satellite missions of the cooperating of Space Agencies that promote, through the Environmental Civil Protection networks, a platform for collaboration and cross-border support in crisis situations. Furthermore, the Copernicus program, coordinated and managed by the European Commission, was created in 2008 to put in practice the European Union's efforts to monitor the Earth and its ecosystems, while guaranteeing citizens preparation and protection in the event of natural or anthropic crisis. The Copernicus program makes available a huge amount of information about our planet, in a complete, open and free way to citizens, public and government authorities, scientists, entrepreneurs and businesses. Copernicus services are based on information from a constellation of dedicated satellites, called "Sentinels", and from dozens of additional satellites, the socalled "participating missions". Specifically, the Copernicus Emergency Mapping Service provides digital maps (reference maps, delineation maps, grading maps) and vector files that refer to the pre and post-event situation. The type of available certified products varies according to the type of calamitous event that occurred and can quickly provide an estimate of the impact and order of magnitude of the damage caused by the event. Thus, based on the type of the calamitous event, the resulting product includes an assessment of the extent, type and importance of specific damage. With particular reference to the 2016 earthquake in central Italy, the Copernicus EMS was immediately activated by the Italian Civil Protection and by the
Italian Space Agency (ASI), thus allowing to start the territorial analysis activities after a few hours using images acquired with optical systems and satellite radars; this allowed for an immediate response to the request. Civil Protection took full advantage of the possibilities offered by Copernicus EMS and more than 100 maps of the interested areas were produced, fully drawing on all the existing data available on social media to obtain the "most reliable" information in the shortest possible time. Finally, we mention the important role played by the Italian constellation of COSMO-SkyMed satellites, consisting of four satellites equipped with radar, whose data were used to quickly assess the extent and impact of damages, by carrying out high precision data and taking responsibility for detecting changes between the two subsequent seismic events (Santoro, 2017). Figure 1 shows a classification map of the damages referred to Arquata del Tronto with the number of destroyed damaged buildings in each cell of a regular grid, including populations, roads, hospitals, collection area, etc.

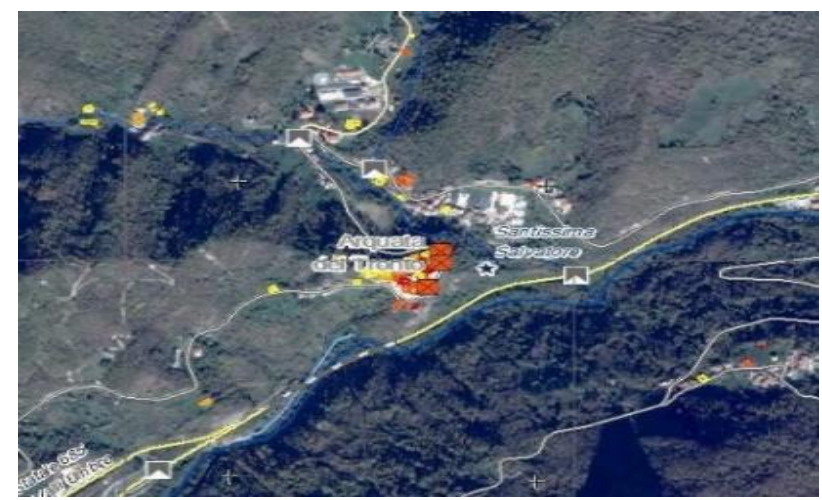

Figure 1. Grading map Arquate del Tronto after earthquake dated 30-10.2016

\section{STATE OF THE ART PLATFORMS OF UNMANNED AERIAL VEHICLES (UAVS)}

In the post-seismic period investigations with classic Geomatics tools are complicated and complex due to residual dangers in the area. The instruments of aerial satellite photogrammetry, such as those described above, are certainly less dangerous but do not produce detailed information on damaged structures, especially for what concerns elevations and facades or interiors mainly due to accessibility problems. To achieve this latter purpose, it is therefore more advantageous, for several reasons, to use an Unmanned Aerial Vehicle (UAV). A UAV aircraft, remotely piloted from a ground station, with low altitude flight, equipped with inertial platform, GPS and RGB, multispectral and thermal cameras for photogrammetric sockets, represents the current frontier for the investigation of the territory. The possibility of installing different sensors makes UAVs very useful for several applications both in urban and rural areas, e.g. for the analysis of the environmental risk or for estimating changes in land use. Many of the difficulties are related to morphological and architectural accessibility, typical problems of post-disaster scenarios. In particular, in the field of Civil Protection where the priority is operator safety, these platforms are used for the observation and collection of data in areas struck by earthquakes, landslides, subsidence and avalanche phenomena, as well as for the control of forests and the prevention of summer fires (Baiocchi et al, 2014). From the operational point of view the potential of these systems have 
been initially tested in Italy to estimate damages in the historic center of L'Aquila caused by the earthquake of April 2009. Currently the research on UAVs involves studies related to specific aspects such as: performance optimization, flight and navigation control, route planning and obstacle avoidance. For example, for what concerns the optimization of the UAV performances, the length of the flight time, necessary to travel waypoints (reference points in the physical space used for navigation), has been minimized in some UAV models by using an approach based on the Travelling Salesman Problem (TSP), the simplest among the tasks of distribution logistics (Erbil et al. 2013). Moreover, there are several options to increase the autonomy of unmanned aerial systems, i.e. their flight length. One is to use combustion engines, even if this choice has the drawback of high purchase, operation and maintenance costs. Furthermore, combustion engines usually produces vibrations which must be isolated from the joint of the cameras and acquisition sensors to obtain images of sufficient quality. The alternative option to increase UAVs' autonomy is to use solar panels assembled on the vehicle / drone wings so as to supply energy to the electric motors. The type of power supply of fixed wing UAV systems that derives from the combination of electric and solar energy provides a significant increase in flight autonomy compared to vehicles powered by purely electric or combustion energy (González-Jorge et al. 2017). Further researches have contributed to the aspect of monitoring the performances of UAVs; for example, a flight stabilization regulator on board a quadrotor has been developed for several applications (Conte et al. 2008). Similarly, a controller has been designed to regulate the speed and performance of a UAV system in several wind turbulence conditions (Alpen et al. 2009). Other researchers have analyzed a method that integrates three-dimensional (3D) point clouds, two-dimensional (2D) digital camera data and data from an inertial measurement unit (IMU) to establish a precise position and identify the performances of a UAV platform (How et al. 2008). Still a large number of contributions concerns, in particular, route planning and obstacle avoidance to this end; an innovative evolutionary algorithm was used to design an intelligent route planner for autonomous navigation of a UAV (Nikolos et al. 2003). Nowadays, with the growing use of UAV vehicles for civil purposes, such as in archaeology, agriculture, first aid, saving lives, etc. obstacle detection is a fundamental aspect for the navigation of a unmanned aircraft. This aspect becomes even more important when UAVs move at low altitudes or indoors where there are several obstacles. In these situations, automatically detecting and preventing obstacles becomes crucial. Obstacle detection techniques are generally divided in two methods: "sensor-based" and "vision-based". The former requires a data sensor to detect obstacles and various sensors use laser beams, radar and ultra-sounds. However, these sensors are, usually, large, heavy and expensive for use in small UAVs for this reason either, stereo or mono "vision-based" methods are preferred. Differently from mono techniques, stereo ones need to obtain the 3D model of objects. Mono techniques include background and foreground separation methods and methods inspired by the functioning of the human brain. Background and foreground separation methods have low efficiency so that finding obstacles with this method is not always a correct procedure. Brain-inspired methods use a similar technique based on how humans perceive and detect obstacles (Badrloo, Varshosaz, 2017). A large part of the literature concerns the specific theme of the guide which has strong connections to flight planning; in fact, the latter is nothing more than a dynamic process of directing an object towards a certain point that can be stationary or dynamic. For example, several trajectory generation methods for a UAV rotor have been demonstrated in Astrov et al. (2010) and Mellinger et al. (2011). UAV applications for Geomatics are, however, used in areas that may be too dangerous for manned aircraft or for specific investigations. Although high resolution images represent a fundamental tool for the assessment of damages on structures, infrastructures and strategic areas in the event of natural emergencies, (Baiocchi et al, 2014), the effective uses of UAV platforms are still in progress. Actually, they still have to overcome a series of limit actions related mainly to the short battery life, to limited coverage areas, to unexpected events associated with variable atmospheric conditions, to the still limited training of the user pilot and, finally, to current legislations that significantly limits UAVs use in most countries (Fernandez Galarreta et al, 2015). Finally, according to what has been reported by Giordan et al (2017), the use of UAV for the $3 \mathrm{D}$ reconstruction of anthropic structures, in particular for historical sites and monuments, has represented the first applications developed and published in the last decade. The use of these remotely piloted systems is of great help in rescue operations involving multiple types of emergencies. For example, real-time aerial images allow one to record and analyze the global environmental changes caused by typhoons, tsunamis and so on; furthermore, estimated data of new damages useful for emergency rescue can also be obtained (Chou et al. 2010). UAV platforms are also very successful in surveillance-related operations; for this reason they can be used in the prevention of forest fires, in particular for those caused by humans (González-Jorge et al. 2017), or in the conservation and cultural heritage management as happened in the most recent case of protection of S. Agostino Church in Amatrice (Chiabrando et al. 2017).

\section{THE TAR PROTOCOL: TOPOGRAPHY APPLIED TO} RESCUE

The title of this section has been deliberately taken from the name of the Italian protocol TAS which is the acronym of "Topografia Applicata al Soccorso"; its equivalent in english is TAR: Topography Applied to Rescue. A series of seismic events have struck central Italy since $24^{\text {th }}$ August 2016, with the subsequent seismic shock of the $26^{\text {th }}$ and $30^{\text {th }}$ October 2016, of the $18^{\text {th }}$ January 2017. The further issues associated with the natural risk, such as exceptional snowfall, the subsequent avalanches, as the snowfall which struck Rigopiano Hotel, the landslides and the floodings caused by the sudden melting of snow, have made the scenario a rarity among the events that have struck the nation in the last century (Feliziani et al. 2017). In Italy several authorities devoted to rescue and, usually, to the management of the emergencies are involved in a catastrophic event. Among such authorities the national Fire Department, referred to with the acronym CNVVF, plays a relevant role. The response to such a complex and specific event has been very prompt and efficient, not only in relation to the employed staff, but also for the different specializations of CNVVF that have experienced the possibility to integrate the respective operative procedures in a particularly involved real scenario actually impossible to simulate. 


\subsection{The Short Term Countermeasures System (STCS)}

The experiences carried out in seismic scenarios that have struck Italy in the last decade have progressively led to the development of an organizative system for the management and implementation, by qualified units of the CNVVF, of urgent technical countermeasures aimed at dealing with structural problems deriving from emergencies. This system is the Short Term Countermeasures System (STCS) aimed at addressing, in a rational and organized way, the structural criticalities that follow disastrous events, both for the management of the assessment phase and for the implementation of the mitigation (shoring works, removal of dangerous parts, dismantling, demolition, etc.) ensuring full interoperability with other units of the European Civil Protection Mechanism. The STCS system has the objective of organizing and managing structural problems with a technical-specialistic approach, elaborating damage assessments, producing drawings able to support planning and implementation of effective countermeasures to mitigate the risk of collapses. To achieve these objectives, the system is integrated with the other specialized functions of the Fire Department such as: TAR (Topography Applied to Rescue), SAF (Speleo Alpin Fluvial), SAPR (Remote Piloted Aircraft Systems), CDV Photo and Video Documentation Centers.

\subsection{Purposes and interventions of the protocol TAR}

Although interdisciplinary and numerous, the different types of survey that the CNVVF deals with on a daily basis, share a common prerogative: the territory. In this regard, an operational support unit based on topographical techniques and the use of GIS has developed within the CNVVF for years, giving space to a new and innovative activity: Topography Applied to Rescue. The TAR operator gains a high sectorial specialization through a targeted theoretical and practical training and is able to associate cartographic knowledge with the reality of urgent technical assistance, thus ensuring the opportunity for decision support in complex contexts. In addition, the TAR operator is able to radio-localize staff and vehicles located in emergency craters in real time, using advanced software and technologies implemented by the CNVVF (Cuzzocrea, Priori, 2014). The experience gained in this field in recent years has made it possible to understand how the TAR service has a transversal interest in all CNVVF activities, allowing not only for the analysis and delineation of the damage scenarios but also for the support to decisions of the units in charge of rescue operations. The TAR Service pursues objectives aimed at improving the effectiveness and efficiency of emergency services through the integrated use of human and instrumental resources. These are used for the production, analysis and use of geo-referenced data, which can be used to facilitate the search for solutions to complex problems relating to the planning and management of emergencies as well as to the "reporting" of the operations carried out. The TAR function has always been activated in extreme conditions, starting from the earthquake that struck Abruzzo in 2009, to the earthquake in Emilia Romagna and to the emergency associated with the sinking of the Costa Concordia ship in 2012 (Figure 2a), allowing to draw up thematic maps of strong value. For example, in the case of the 2009 earthquake in Abruzzo, the delimitation of inaccessible areas or forbidden roads (Figure $2 b$ ), the mapping of the routes carried out by VVF staff engaged in search for missing persons are only some application cases of the TAR service in emergency area. Furthermore, as part of the national forest fire prevention campaign - AiB 2013, a TAR station was set up at the VVF National Operational Center. In addition to geolocation, it also allowed for the three-dimensional reconstruction of the routes traveled by Canadair aircraft used during the extinguishing operations of forest fires. The TAR teams adopt the national analogue VVF network with service frequencies $73 \mathrm{MHz}$ and $400 \mathrm{MHz}$ for geolocation. Each means of transport and VVF operator is equipped with radio devices, which, in dialogue with the radio bridges located in the area, send data on VVF coordinates to the kits for geolocation of TAR teams.

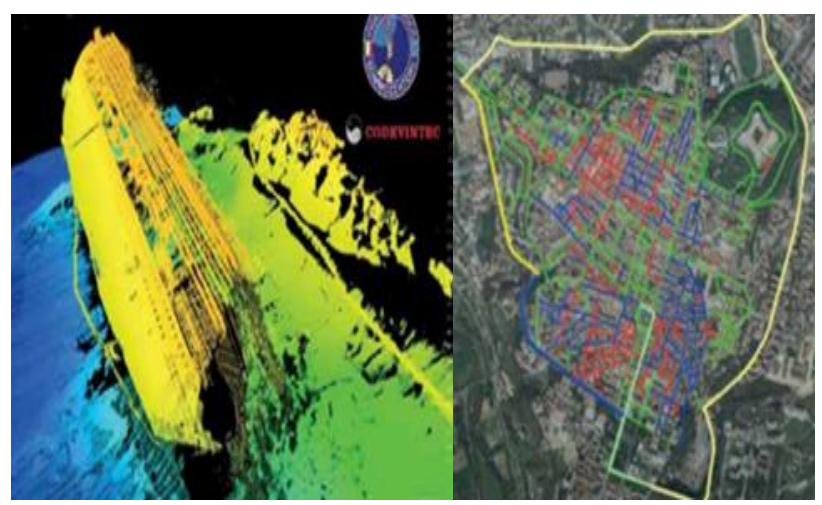

Figure 2. a) Three-dimensional scan of the Costa Concordia ship; b) Cartographic elaboration of the traffic of the center of L'Aquila during the 2009 earthquake emergency.

The TAR operator kit consists essentially of a portable suitcase with a radio having USB interface and software for geolocation and interrogation of radio equipment. The geolocation software transfers data to the GIS Ozi Explorer service, ensuring the TAR service for the control and management of the several units used in the operational scenario, in emergency situations, or in the territory, in the case of ordinary activities. It is fundamental to note that TAR transversality is a consequence of its versatility of use in different contexts. In fact, in addition to the known seismic events, so far there have been numerous types of interventions for which the TAR has been a necessary support tool; some examples are listed below: map of the forest fire, search for missing persons, water rescue in marine, lake and river environment, floods, accidents in industrial sites, mapping of research areas and points of interest (e.g. Costa Concordia ship). It should be emphasized that the mappings drawn up during emergencies from the TAR service allow for the historical database of CNVVF interventions to be implemented; hence it can be consulted after the repetition of cyclic disastrous events for forecasting purposes, or for the dislocation of operational structures. Although the CNVVF TAR organization is well structured and consolidated, initiatives have recently been undertaken with the aim of strengthening the sector. One of the most important is the stipulation of a framework agreement with the Military Geographical Institute (IGM) to acquire digital cartography and create a common database to be shared. Currently the TAR system is able to adapt to any type of CNVVF request: an example in this regard is the diversification of the software used for the mapping of the operating scenarios (Ozi Explorer, ArcGIS, Global Mapper) which allow one to modulate the application of the TAR service according to the level of in-depth analysis of the geographical analysis which is subject of interest.

\section{APPLICATIONS OF UAV SYSTEMS IN DAMAGE SCENARIOS}

During an emergency such as an earthquake, conventional inspection to assess the state of damage to buildings requires special tools, a lot of time and considerable costs. Therefore, 
given the high number of buildings that require safety interventions and rehabilitation measures, effective use of limited resources, such as time and equipment, as well as the safety of the involved personnel are important aspects. The applications shown below are intended to highlight how the aforementioned objectives can be achieved through the use of the tools put forth by Geomatics.

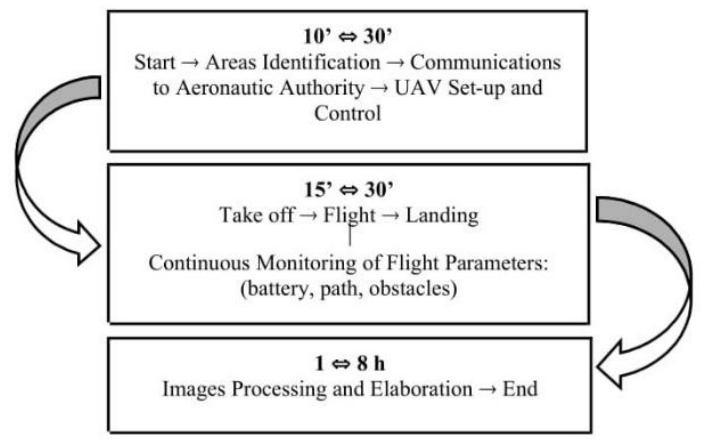

Figure 3. Workflow of a generic flight mission with UAV with relative timing

4.1 Case study 1: context analysis in the municipality of Castelsantangelo sul Nera

This first study case concerned a context analysis of the municipality of Castelsantangelo sul Nera, struck from the earthquakes that have interested central Italy since August 2016, in order to provide the emergency manager and planner with an appropriate and more detailed tool than the maps produced by the Copernicus EMS service. The documents produced in the context analysis allow one to better visualize the area and decide the portions in which it is advisable to intervene in a very short time to avoid further collapses and damage following an aftershock. Castelsantangelo sul Nera is a town in the Marche region of 281 inhabitants in the province of Macerata; in its territory there are the sources of the Nera river and the beginning of the Valnerina valley. This municipality is classified as a high seismicity area (zone 1), as reported in the 2015 seismic classification chart of the Italian territory; it is characterized by a ground acceleration value of $0.225-0.250 \mathrm{~g}$. In particular, one of the 3 strongest shocks of 2016, that of 26 October with a magnitude of 5.9, had Castelsantangelo sul Nera as its epicenter. From a geological point of view, the municipality is located south-east of the fourth quadrant of sheet 132 - Norcia of the Geological Map of Italy in scale 1: 100,000 and consists mainly of limestones and debris of the aquifer. To get images of the damage caused by the earthquake, a drone flight was planned using a SKYROBOTICS SR-SF6 UAV platform equipped with a DSC-QX100 camera, GNSS system and inertial base for settling the vehicle in motion. The flight was designed to cover the entire area of the municipality, giving a general overview of the damage to be provided to the emergency manager. Figure 3 shows an example of work flow and processing of the images acquired for a generic flight mission. In addition, a topographic support network with 13 materialized points, placed on the ground with the function of GCPs - Ground Control Points, was created through measurements with GNSS (Global Navigation Satellite System) and total station systems. All markers have been georeferenced in the common reference system WGS 84 / UTM fused 33N. Subsequently, the flight plan was designed in order to cover the whole municipality. This phase is closely related to the technical features required by the products of the final map, especially in terms of spatial resolution and overlap percentages between images, to ensure appropriate 3D photogrammetric processing. UAV systems include ad hoc software to semiautomatically plan flights and acquisitions to be loaded on the autopilot system. It has also to be emphasized that, unlike ordinary situations, in an emergency scenario there is no possibility of waiting for perfect weather conditions for the sockets, except for the minimum flight safety ones. During the flight, whose characteristics are shown in Table 1, 681 images were acquired with a resolution of $5472 \times 3648$ pixels. Figure 4 shows some examples of raw images acquired through the UAV platform. Once the images have been acquired, they must be properly processed to generate products capable of documenting the geometric and thematic perspective of the surveyed area; these products are sparse clouds, dense point clouds, 3D models, DEM - Digital Elevation Model and orthophotos. The preliminary phases involve alignment of the images, with the search for homologous points, the identification of the control points and the import of their coordinates, the camera calibration for the correction of optical distortions and the construction of the mesh.

\begin{tabular}{|c|c|}
\hline UAV platform: & SKYROBOTICS \\
\hline Room model: & DSC-QX100 \\
\hline Average flight altitude: & $120 \mathrm{~m}$ \\
\hline Pixel size: & $2.44 \times 2.44 \mu \mathrm{m}$ \\
\hline $\mathrm{N}^{\circ}$ of images: & 681 \\
\hline Overlooked area: & $0.343 \mathrm{~km}^{2}$ \\
\hline Ground resolution: & $2.61 \mathrm{~cm} / \mathrm{pixel}$ \\
\hline
\end{tabular}

Table 1. Characteristics of the flight mission

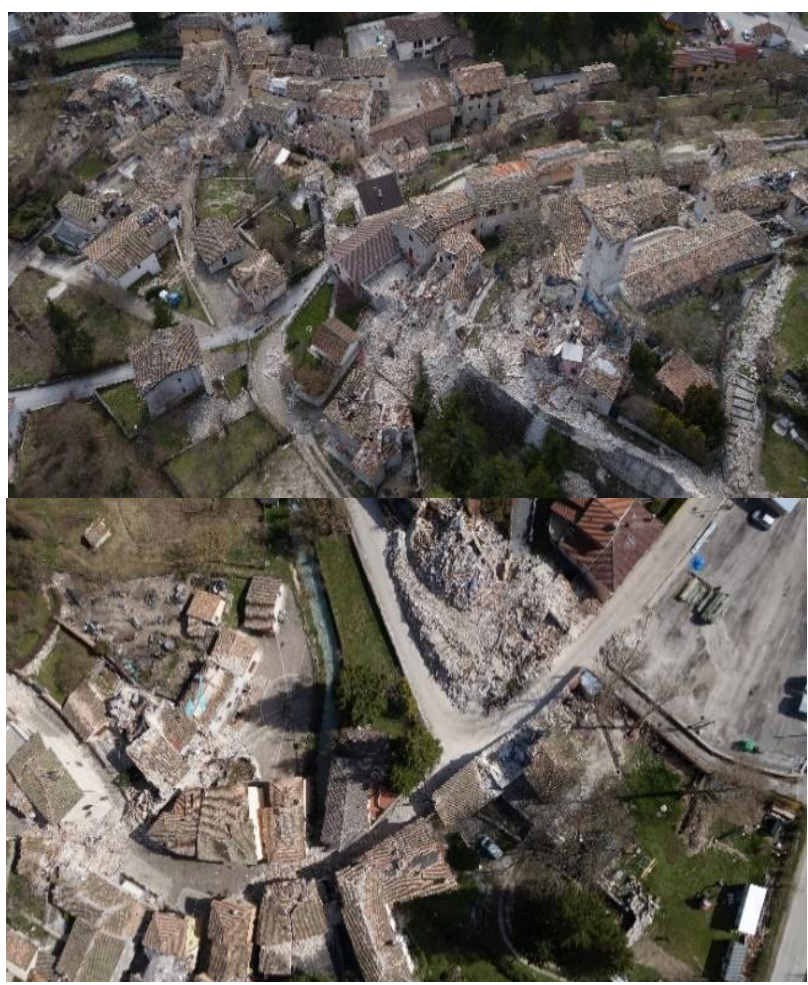

Figure 4. Images by UAV of Castelsantangelo sul Nera

The bundle adjustment phase ensures the accuracy of the 3D model. The software used is AGISOFT PHOTOSCAN PROFESSIONAL version 1.3.3. The processing products are listed in the sequel. 


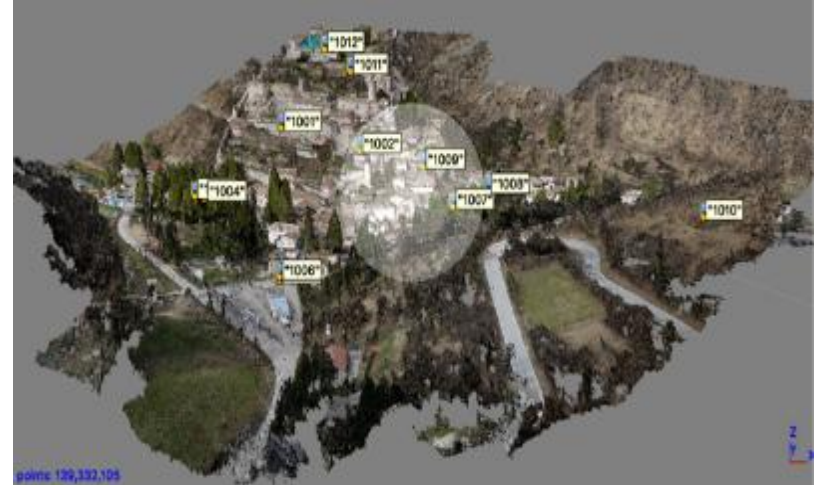

Figure 5. Dense point cloud of Castelsantangelo sul Nera

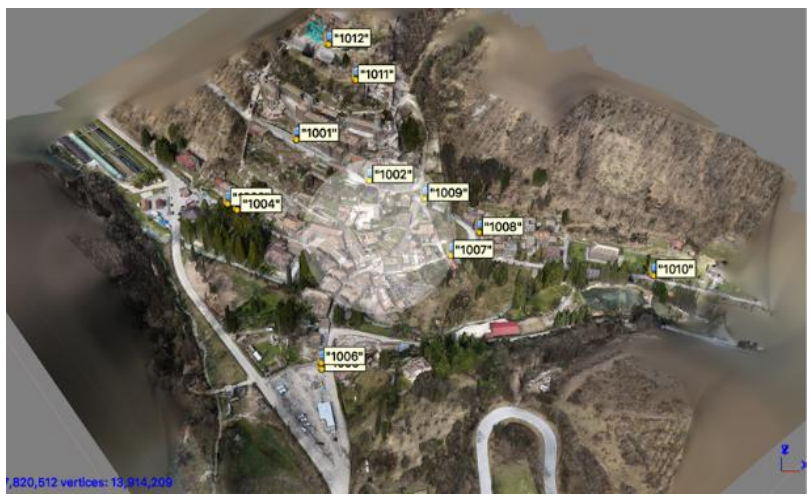

Figure 6. 3D Model of Castelsantangelo sul Nera

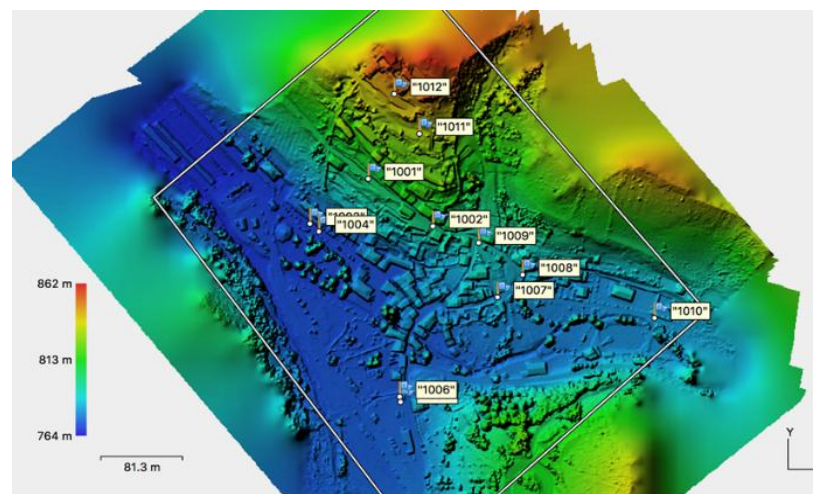

Figure 7. DEM of Castelsantangelo sul Nera

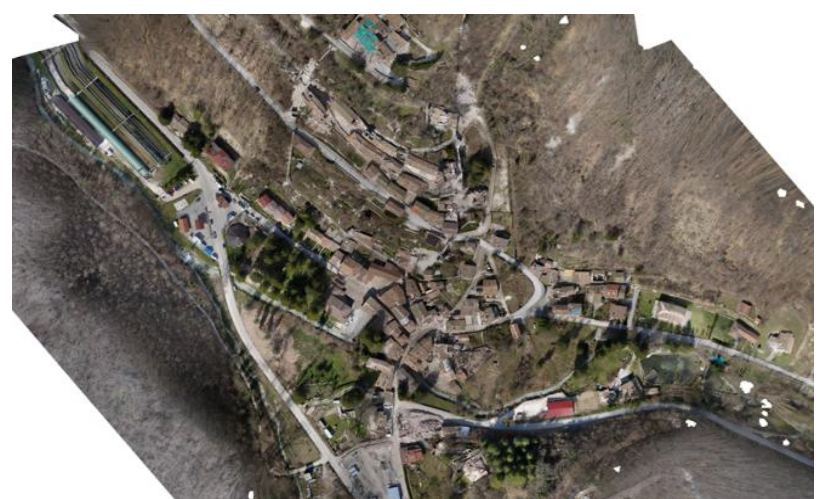

Figure 8. Orthophoto by UAV on Castelsantangelo sul Nera
As it can be seen from Table 2, the total accuracies and those of each coordinate of the control points are centimetric. The reconstruction that has been made of the entire area of the municipality of Castelsantangelo sul Nera could also be repeated for other locations, allowing for a context analysis of the area struck by the earthquake.

\begin{tabular}{|l|l|l|l|l|l|}
\hline Label & X error $\mathbf{( c m})$ & Y error $\mathbf{( c m )}$ & $\mathbf{Z}$ error $\mathbf{( c m )}$ & Total $\mathbf{( c m )}$ & Image (pix) \\
\hline$" 1001 "$ & 1.17011 & -1.41066 & -0.148784 & 1.83883 & $2.124(5)$ \\
\hline$" 1003 "$ & -4.33688 & 1.36635 & -2.43909 & 5.1599 & $0.000(1)$ \\
\hline$" 1004 "$ & -1.46093 & 1.27588 & -0.563803 & 2.01992 & $0.262(6)$ \\
\hline$" 1005 "$ & 0.36425 & -0.227575 & 0.403163 & 0.589074 & $0.006(6)$ \\
\hline$" 1008 "$ & -0.063817 & 0.497438 & 0.149384 & 0.52329 & $0.003(6)$ \\
\hline Total & $\mathbf{2 . 1 1 8 9}$ & $\mathbf{1 . 0 7 5 5 5}$ & $\mathbf{1 . 1 3 7 8 9}$ & $\mathbf{2 . 6 3 4 6 5}$ & $\mathbf{0 . 9 7 8}$ \\
\hline
\end{tabular}

Table 2 - Accuracies of some GCPs

This analysis is of fundamental support for the choice of the best intervention strategy to be adopted in the entire area, thus allowing the authorities in charge of managing the emergency to dispose of an updated situation from multiple perspectives. In this application case, the entire area of the village has been reconstructed and the products obtained from image processing can be used for several activities. Among these we mention planning of the choices to be taken to counteract the most urgent dangers, in order to prevent further damage following subsequent aftershocks, the estimate of the rubble to be moved and removed, the study of the viability and additional aspects that will be dealt with in case study 2. A product of particular interest in a context analysis is the DEM obtained by analyzing images as a partial reworking of the dense cloud. Its main operational applications concern the calculation of volumes / dimensions and the study of the viability and practicability of the roads, both aspects of paramount importance in a postearthquake analysis. Generally in 6-8 hours it is possible to obtain precise and accurate images with a standard deviation of 2-3 cm from a comparison with the GCPs. We report in the sequel a graph taken from the literature that shows an approximate attempt to allocate time in a typical photogrammetric workflow (Nex and Remondino, 2014); it can be deduced that the most time-consuming phase is that associated with the construction of a dense point cloud. In the case study discussed here, it required a processing time of approximately 6 hours.

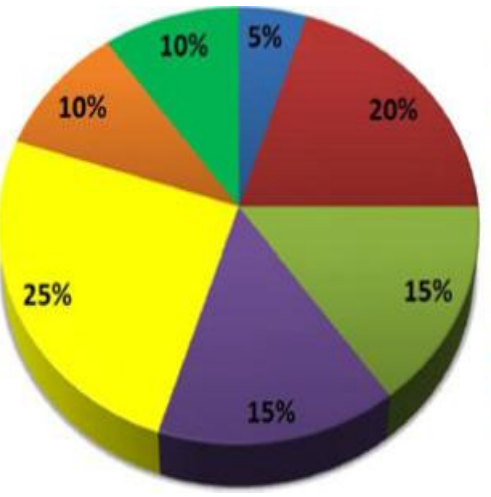

: Flight planning

- Image acquisition

r.r. GCPs field measurement

- Image triangulation

$\checkmark$ DSM generation

Ortho / Mosaicking

Geature extraction

Figure 9. Timing of a typical photogrammetric workflow

4.2 Case study 2: detail analysis in the municipality of Lacco Ameno (Ischia island)

In this second study case, a detailed analysis was performed using images acquired by UAV on a single structure located in the municipality of Lacco Ameno in the island of Ischia. The 
purpose of this type of analysis is to fill in the form called TriageEdEm (Building Triage in the emergency) that the Fire Brigade is required to draw up during its rescue activities to evaluate the interventions to be carried out on a damaged structure. This activity is an innovation in the construction field, since this technique has been used so far by the Fire Brigade only to carry out analyses on cultural heritage assets and on particularly high structures. The object of our study was a residential structure, located in the municipality of Lacco Ameno in the island of Ischia, damaged by the earthquake of 21 August 2017. The TriageEdEm sheet aims to rapidly classify the consequences induced by an adverse event on a structure or building in terms of safety. The classification, functional to subsequent actions and countermeasures, takes place through an expeditious assessment and classification of critical issues for the safety of people and the identification of the rapid interventions necessary for their control or removal. The critical issues are analyzed with reference to the context, the proximity area and the interior spaces. Data to be reported in the TriageEdEm sheet are the reference data related to the applicant, the identification name of the building, the starting date and time of the inspection and the GPS - WGS84 coordinates with the address of the structure to be geolocated. Subsequently, one has to fill in a field concerning the geometry and construction features of the building, schematize the plan shape of the building (indicating the point of acquisition of the GPS coordinates and the North direction), approximately indicate the height and plan dimensions of the building, the type of construction, the structural type and the relevance of the building. Furthermore, it is necessary to carry out a damage assessment check-list with the aim of verifying the presence of possible falls or collapses of structural and non-structural elements, or the presence of other critical issues that may represent a danger for the safety of people (e.g. fall of heavy and / or blunt elements, release of dangerous substances, etc.) by carrying out an analysis of the scenario through 3 steps:

1) context analysis, aimed at highlighting and characterizing the possible presence of critical issues affecting the construction under assessment;

2) proximity analysis, aimed at highlighting and characterizing the possible presence of critical issues induced by the construction in its proximity area. The elements that are evaluated are the vertical elements, the roof and the base. Furthermore, the possible presence of critical issues related to external systems must be checked as well as the presence of failures with or without the potential presence of falls and / or collapses with reference to the facades, external walls, roof and basements;

3) internal analysis, aimed at highlighting and characterizing the possible presence of critical issues induced by the construction in its internal area. The elements that are evaluated, both of structural and non-structural nature, are the internal walls, the horizontal elements, the movable elements and the stairs. Furthermore, the presence of critical issues related to internal installations must be ascertained and the presence of failures with or without the potential presence of falls and / or collapses with reference to internal walls, floors, stairs, furnishings, mobile elements and systems must be analyzed. Whenever is possible to eliminate all critical issues through rapid interventions, the situation must be judged to be readily recoverable and the necessary rapid interventions must be reported in the EI Needs. The TriageEdEm form also indicates: the already adopted urgent measures, the tools and resources necessary to carry out the type of indicated intervention, the needs of ordinary and special temporary works, any repercussions on the viability, on the activities or on the adjacent buildings determined by the damage scenario of the building, the priority indicators with the relative level.

All fields of the TriagEdEm sheet have been filled in, the evaluation of the construction comes out as a summary judgment to be reported also within a summary graphic symbol. With specific reference to this case, a flight was carried out by using a vehicle from the fleet of the SAPR core of the CNVVF, in particular, a remote-controlled, multi-rotor system called DJI Inspire 1 whose characteristics of which are shown in Table 3.

\begin{tabular}{|c|c|}
\hline UAV platform: & DJI Inspire 1 \\
\hline Room model: & Zenmuse X5 \\
\hline Average flight altitude: & $120 \mathrm{~m}$ \\
\hline Pixel size: & $3.76 \times 3.76 \mu \mathrm{m}$ \\
\hline $\mathrm{N}^{\circ}$ of images: & 101 \\
\hline Overlooked area: & $1480 \mathrm{~m}^{2}$ \\
\hline Ground resolution: & $3-5 \mathrm{~cm} / \mathrm{pixel}$ \\
\hline
\end{tabular}

Table 3. Characteristics of the flight mission

The aircraft sailed at an average speed of $5 \mathrm{~m} / \mathrm{sec}$ above the structure located in Borbonica street $\mathrm{n}^{\circ} 10$, for a duration of about 13 minutes, acquiring nadiral and lateral images. The Figure 10 shows some examples of images of damage to buildings acquired through the used UAV platform in Borbonica street n.10.
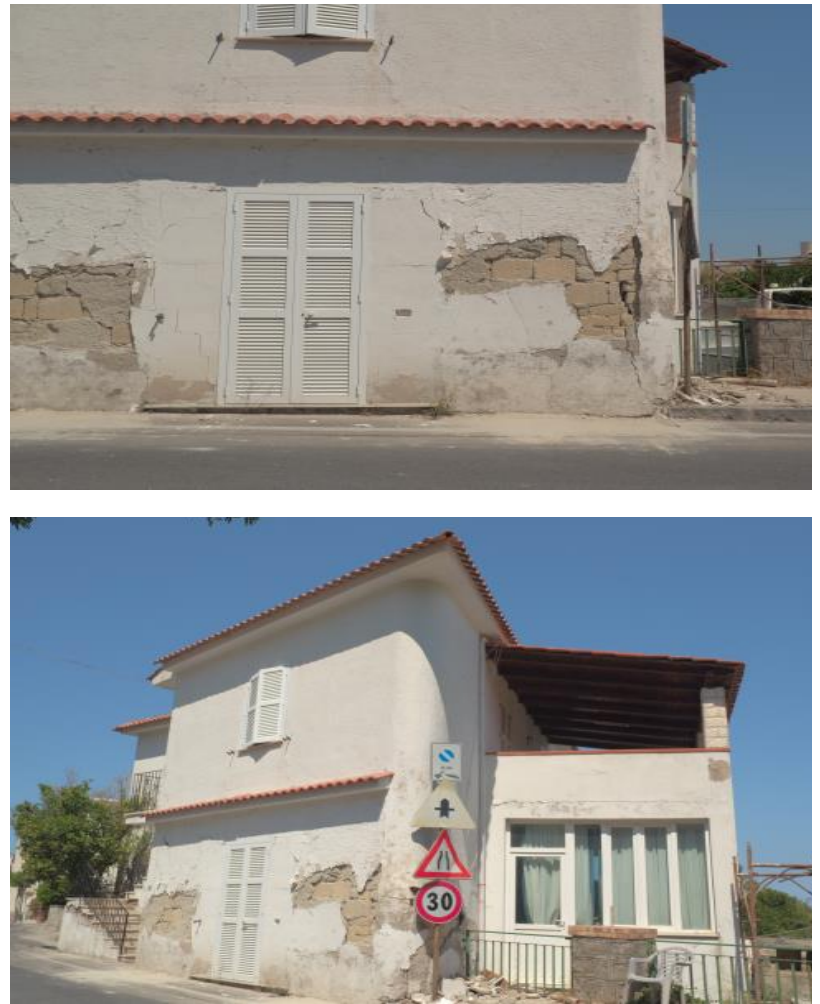

Figure 10. Images of damages by UAV of a building in Lacco Ameno

The processing of the images took place in a similar way to what was previously described, except for what concerns the GCPs.Actually, it was not realized in this case study due to time and viability, since it has not been possible to close an entire 
portion of a road. main used for emergency operations. The processing products useful to complete the TriageEdEm card are shown in the following features.

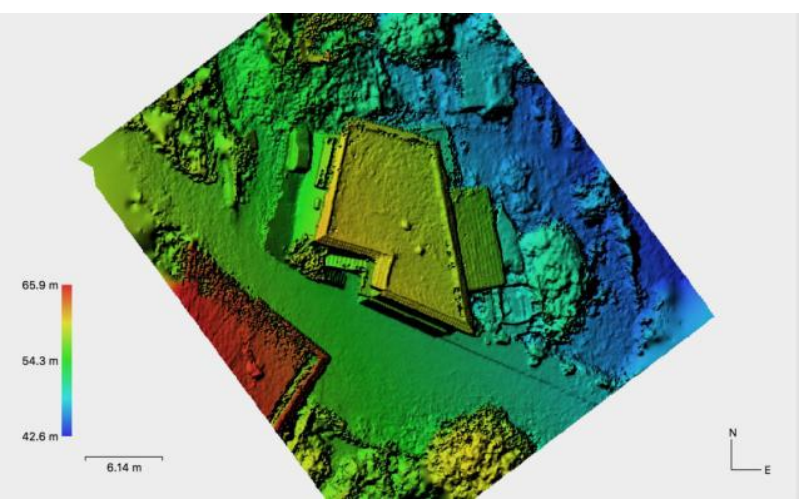

Figure 11. DEM of a building in Lacco Ameno

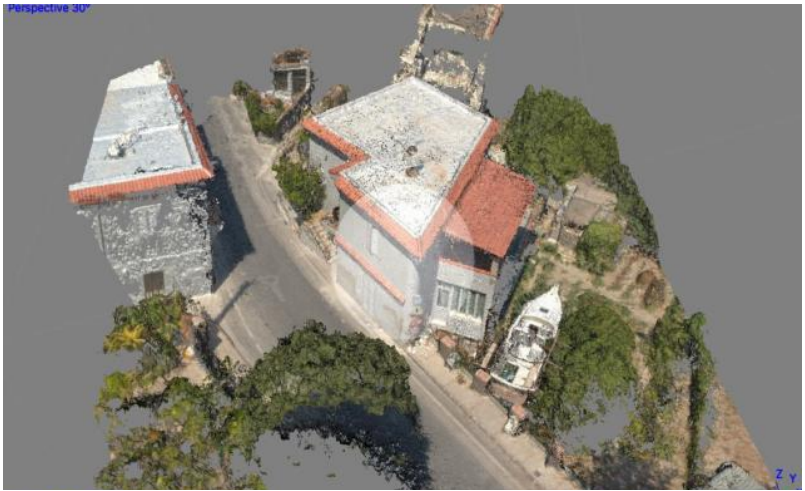

Figure 12.3D Model of a building in Lacco Ameno

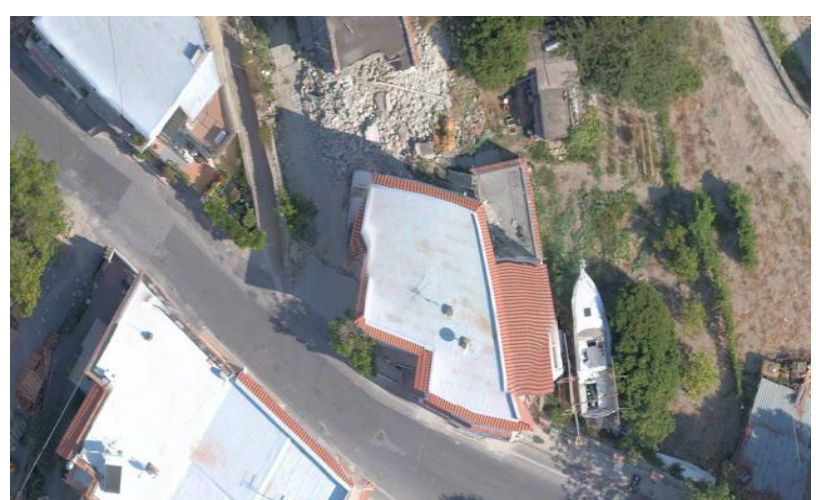

Figure 13. Orthomosaic of a building in Lacco Ameno

The accuracy of the products is in the order of tens of centimeters. Although this value does not represent a high precision measure, it is nevertheless acceptable and sufficient in many emergency applications. However, if the use of UAV platforms to acquire data relating to post-earthquake damage, should become a standard procedure for the compilation of the form, it would be desirable to install an adequate topographic support network that could guarantee greater precision in the measurement of the damage to buildings. These factsheets are intended as a synthesis between the technical-scientific research and the consolidated experience of the Fire Brigade and have been drawn up as part of the activities related to the management of the post-earthquake emergency in the area struck by the L'Aquila earthquake.
Figure 14 shows the TriageEdEm sheet compiled by the Fire Brigade for the structure of case study 2, with the help of the STOP Vademecum sheets - Technical sheets for temporary works for post-earthquake safety. The results from the compilation of the TriageEdEm form in the case of the Lacco Ameno structure are briefly presented in terms of criticality judgments and choice of the type of intervention to be implemented. TheTriageEdEm card in Figure 14 describes a building, illustrated in the case study 2 , predominantly damaged in the lower section, with evident cracks and crashes of perimetral masonry and with significant damages on the facade across the street and on the externally inaccessible staircase.

The results of the TriageEdEm are summarized in the sheet reported in Figure 15.

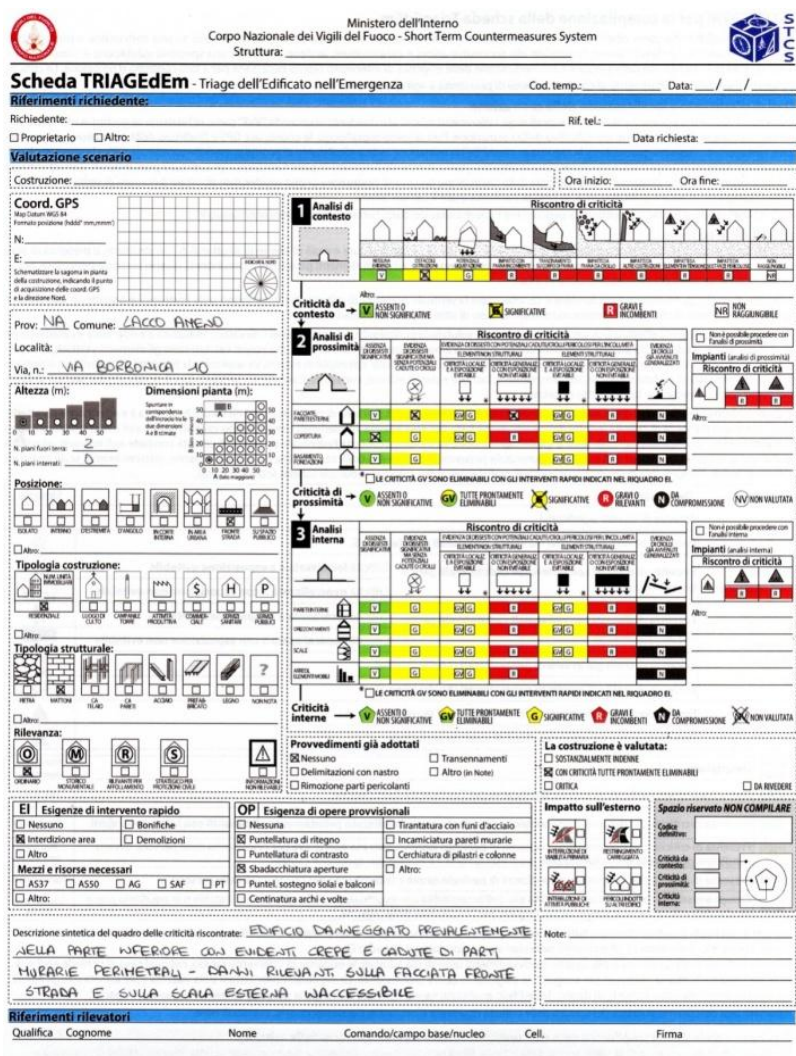

Figure 14. TriageEdEm card of a building in Lacco Ameno

\begin{tabular}{|l|l|}
\hline Context analysis: & $\begin{array}{l}\text { SIGNIFICANT critical } \\
\text { issues }\end{array}$ \\
\hline Proximity analysis: & $\begin{array}{l}\text { SIGNIFICANT critical } \\
\text { issues }\end{array}$ \\
\hline Internal analysis: & $\begin{array}{l}\text { Critical issues NOT } \\
\text { ASSESSED }\end{array}$ \\
\hline Adopted measures: & Nothing \\
\hline Construction Rating: & $\begin{array}{l}\text { With critical issues READY } \\
\text { TO BE ELIMINATED }\end{array}$ \\
\hline Rapid intervention needs: & AREA INTERDICTION \\
\hline $\begin{array}{l}\text { Requirements for temporary } \\
\text { works: }\end{array}$ & $\begin{array}{l}\bullet \text { Shoring up } \\
\bullet\end{array}$ \\
\hline
\end{tabular}

Figure 15. Synthesis of TriageEdEm card

Generally the consolidation interventions, indicated by the TriageEdEm card, consisted in temporary works of securing represented by the bridge support structures, realized with joint pipes that allow for the containment of the buildings and the 
vehicular and pedestrian passage, otherwise threatened by damaged buildings, or by tunnels with joint pipes to protect the road section, as represented in the Figures 16 and 17.

Other typologies of interventions indicated by the Triage card, expect the securing of the building artifacts through steel ties rod and wooden planking as well as wooden yawnings of the compartments (Figures 18-19)

In any case please note that these temporary works are not alternative to the structural consolidation interventions on the buildings.

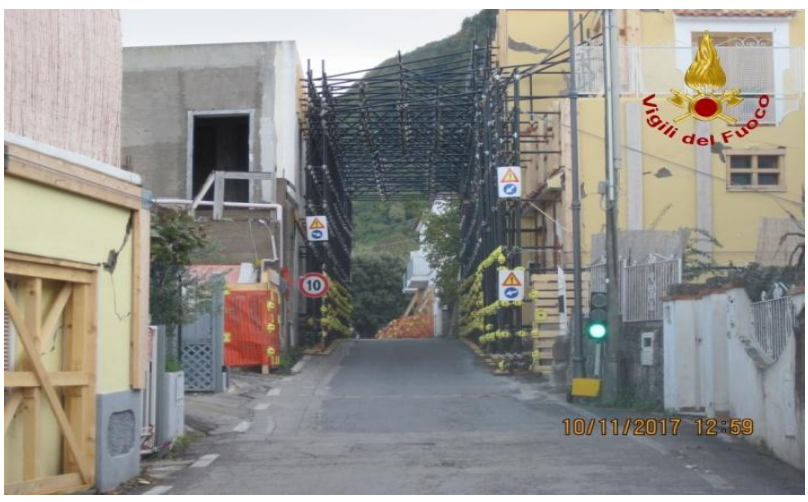

Figure 16. Bridge support structures

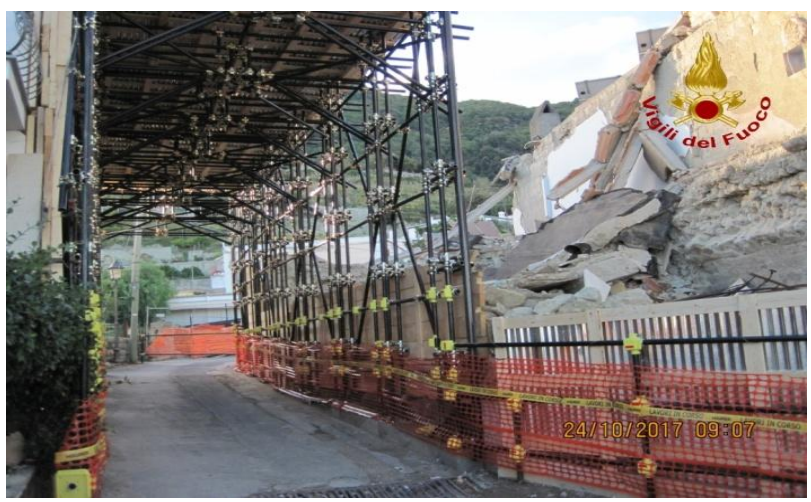

Figure 17. Tunnel with joint pipes

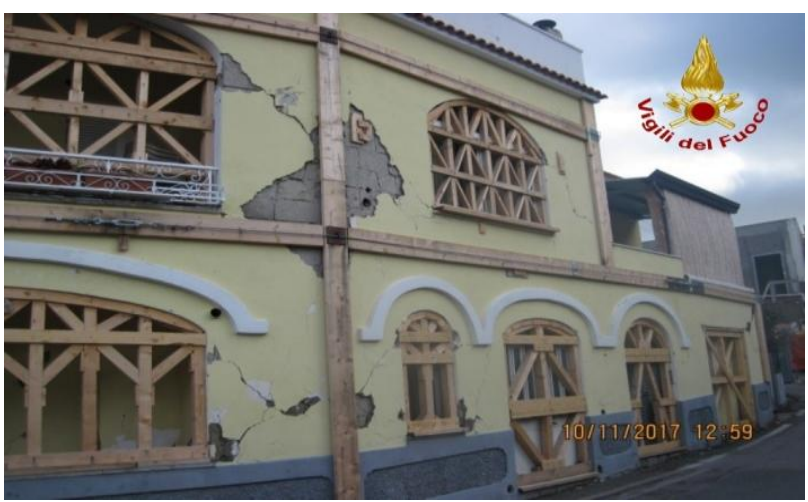

Figure 18. Examples of wooden planking for windows

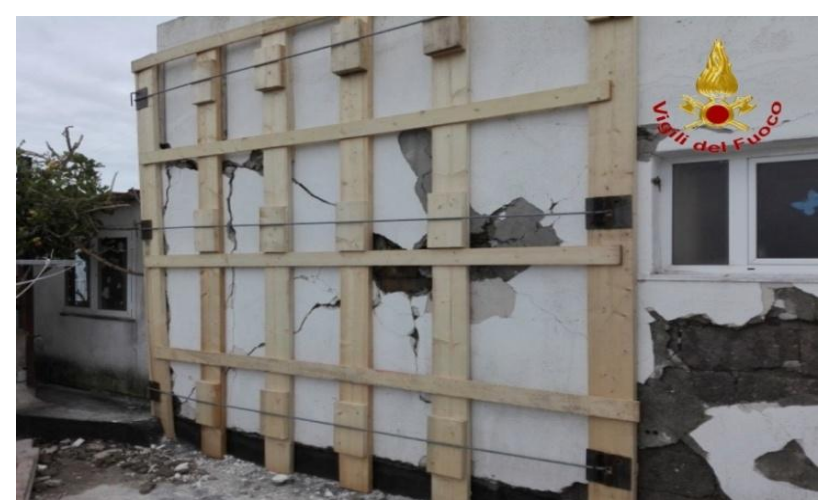

Figure 19. Examples of wooden planking for walls

\section{CONCLUSIONS}

It is important to underline that an aspect of significant importance connected to the use of UAV platforms in emergency situations is computational time. Until now, UAV platforms have been used exclusively to acquire images of buildings belonging to the cultural heritage or of high-rise buildings. On the contrary they could also be used for ordinary structures, as shown in this study case. The advantages of the latter application can be summarized in two main points, namely reduction of rescue times and increase in operator safety. As regards the first aspect, currently the Triage activity, supported by inspections with in situ characterization of the damage of the buildings, takes a long time, since it takes place manually by a few specialized operators, in conditions of poor traffic, surrounded by rubble and in extreme operating conditions. Viceversa, the detection through the use of remotely piloted systems would lead to a reduction in the time required for the quantification of building damage, with a consequent advantage in the choice of the rehabilitative interventions. From the point of view of operator safety, currently the Triage activity sees operators risking their lives in dangerous scenarios where their competence is needed to assess the state of damage and take decisions on subsequent interventions to be carried out. On the contrary the 3D models of the building, processed by the sets of images detected by the UAV platforms, allow the operators to evaluate the damage on the structures, guaranteeing them a much greater safety.

\section{ACKNOWLEDGEMENTS}

This work has been carried out under the GAMHer project: Geomatics Data Acquisition and Management for Landscape and Built Heritage in a European Perspective, PRIN: Progetti di Ricerca di Rilevante Interesse Nazionale - Bando 2015, Prot. 2015HJLS7E GAMHer Website: https://site.unibo.it/gamher/en

\section{REFERENCES}

Alpen M., Frick K., Horn J. (2009). Nonlinear modeling and position control of an industrial quadrotor with on-board attitude control. Proceedings of the IEEE International Conference on Control and Automation, 2329-2334

Astrov I., Pedai A., Rustern E. (2010). Desired trajectory generation of a quadrotor helicopter using hybrid control for enhanced situational awareness. Proceedings of the IEEE International Conference on Information and Automation (ICIA) Shanghai, 1003-1007

Badrloo S., Varshozas M. (2017). Vision based obstacle detection in UAV imaging. The International Archives of the 
Photogrammetry, Remote Sensing and Spatial Information Sciences, $21-25$

Baiocchi V., Dominici D., Milone M. V., Mormile M. (2014). Development of a software to optimize and plan the acquisitions from UAV and a first application in a post-seismic environment European Journal of Remote Sensing, 47 (1), 477-496

Chiabrando F., Di Lolli A., Patrucco G., Spano' A., Sammartano G., Teppati Lose' L. (2017). Multitemporal 3D modeling for cultural heritage emergency during seismic events: damage assessment of S. Agostino church in Amatrice (RI). The International Archives of the Photogrammetry, Remote Sensing and Spatial Information Sciences, Volume XLII-5/W1 GEOMATICS \& RESTORATION - Conservation of Cultural Heritage in the Digital Era, 22-24 May 2017, Florence, Italy, 69-76

Chou T., Yeh M., Chen Y., (2010). Disaster monitoring and management by the unmanned aerial vehicle technology. ISPRS Journal of Photogrammetry and Remote Sensing, 38, 137-142

Conte G., Doherty P. (2008). An integrated UAV navigation system based on aerial image matching. Proceedings of the IEEE Aerospace Conference, 1-10

Cuzzocrea F., Priori F. (2014). Le tecniche topografiche applicate al soccorso. GEOmedia, 18 (3), 10-14

D'Urso M.G., Masi D., Zuccaro G., De Gregorio D. (2018). Multicriteria Fuzzy Analysis for a GIS -based Management of Earthquake Scenarios COMPUTER AIDED CIVIL AND INFRASTRUCTURES ENGINEERING, 33 (2018) 165-179, doi: $10.1111 /$ mice. 12335

Erbil M.A., Prior S.D., Keane A.J. (2013). Design Optimisation of a Reconfigurable Perching Element for Vertical Take-Off and Landing Unmanned Aerial Vehicles. International Journal of Micro Air Vehicles, 5, 207-228

Feliziani F., Lorusso O., Ricci A., Massabo' A., Di Lolli A., Colangeli A., Fiorini M. (2017). Modelli 2D e 3D in scenari emergenziali: impieghi operativi di UAV per la pianificazione delle attività di ricerca e soccorso e per le valutazioni preliminari su edifici ad elevato sviluppo verticale. UAV \& SAPR: using drones in rescue operation. Roma, 29 Marzo 2017

Fernandez Galarreta J., Kerle N., Gerke M. (2015). UAV-based urban structural damage assessment using object-based image analysis and semantic reasoning. Natural Hazard and Earth System Sciences, 15, 1087-1101

Giardino M., Perotti L., Lanfranco M., Perrone G. (2012). GIS and geomatics for disaster management and emergency relief: a proactive response to natural hazards. Applied Geomatics, 4, $33-45$

Giordan D., Manconi A., Remondino F., Nex F. (2017). Use of unmanned aerial vehicles in monitoring application and management of natural hazards. Geomatics, Natural Hazards and Risk

Gonzales-Jorge H., Bueno M., Martinez-Sanchez J., Arias P. (2017). Low-altitude long-endurance solar unmanned plane for forest fire prevention: application to the natural park of Serra do Xures (Spain). The International Archives of the Photogrammetry, Remote Sensing and Spatial Information Sciences, 135-139
How J.P., Bethke B., Frank A., Dale D., Vian J. (2008). Realtime indoor autonomous vehicle test environment. Transactions on IEEE Control System, 51-64

Mellinger D., Kumar V. (2011). Minimum snap trajectory generation and control for quadrotors. Proceedings of the IEEE International Conference on Robotics and Automation (ICRA) Shanghai, 2520-2525

Nikolos I.K., Valavanis K.P., Tsourveloudis N.C., Kostaras A.N. (2003). Evolutionary algorithm based offline/online path planner for UAV navigation. IEEE transaction on System, Man, and Cybernetics, 898-912

Santoro E. (2017). The acquisition, production and dissemination of geospatial data for emergency management and preservation of cultural heritage. The International Archives of the Photogrammetry, Remote Sensing and Spatial Information Sciences, Volume XLII-5/W1 - GEOMATICS \& RESTORATION - Conservation of Cultural Heritage in the Digital Era, 22-24 May 2017, Florence, Italy, 15-24 